\title{
Clinical and histopathological features of acute kidney injury in adult-onset minimal change nephrotic syndrome
}

Kozo Nishide, Shinya Nakatani, Katsuhito Mori, Fumiyuki

Morioka, Yuri Machiba, Hideki Uedono, Akihiro Tsuda, Masaaki

Inaba, Eiji Ishimura \& Masanori Emoto

\begin{tabular}{|c|c|}
\hline Citation & Clinical and Experimental Nephrology. 25(3); 261-269. \\
\hline Issue Date & 2021-03 \\
\hline Published & $2020-11-28$ \\
\hline Type & Journal Article \\
\hline Textversion & Author \\
\hline $\begin{array}{c}\text { Supplementary } \\
\text { material }\end{array}$ & $\begin{array}{l}\text { Electronic supplementary material is available at } \\
\text { https://doi.org/10.1007/s10157-020-01992-8. }\end{array}$ \\
\hline Rights & $\begin{array}{l}\text { This is a post-peer-review, pre-copyedit version of an article published in Clinical } \\
\text { and Experimental Nephrology. The final authenticated version is available online } \\
\text { at: https://doi.org/10.1007/s10157-020-01992-8. } \\
\text { See Springer Nature terms of use. } \\
\text { https://www.springer.com/gp/open-access/publication-policies/aam-terms-of-use }\end{array}$ \\
\hline DOI & $10.1007 / \mathrm{s} 10157-020-01992-8$ \\
\hline
\end{tabular}

Self-Archiving by Author(s)

Placed on: Osaka City University Repository

Nishide, K., Nakatani, S., Mori, K. et al. Clinical and histopathological features of acute kidney injury in adult-onset minimal change nephrotic syndrome. Clinical and Experimental Nephrology. 25, 261-269. (2020). https://doi.org/10.1007/s10157-020-01992-8 


\section{Clinical and Histopathological Features of Acute Kidney Injury in Adult-onset Minimal Change Nephrotic Syndrome}

Kozo Nishide ${ }^{1}$, Shinya Nakatani ${ }^{1}$, Katsuhito Mori ${ }^{2}$, Fumiyuki Morioka ${ }^{1}$, Yuri Machiba ${ }^{1}$, Hideki

Uedono $^{1}$, Akihiro Tsuda ${ }^{1}$, Masaaki Inaba ${ }^{1,3}$, Eiji Ishimura ${ }^{2,4}$, and Masanori Emoto ${ }^{1,2}$

${ }^{1}$ Department of Metabolism, Endocrinology and Molecular Medicine, Osaka City University

Graduate School of Medicine, 1-4-3 Asahi-machi, Abeno-ku, Osaka, 545-8585, Japan

${ }^{2}$ Department of Nephrology, Osaka City University Graduate School of Medicine, 1-4-3 Asahimachi, Abeno-ku, Osaka, 545-8585, Japan

${ }^{3}$ Department of Internal Medicine, Ohno Memorial Hospital, 1-26-10 Minamihorie, Nishi-ku, Osaka, 550-0015, Japan

${ }^{4}$ Department of Nephrology, Meijibashi Hospital, 1-358-3 Miyakenishi, Matsubara, Osaka, 580-

0045, Japan

Word count: Title, 14 words; Abstract, 234 words; Main body, 4064 words

Table and figures: 4 tables, 2 figures, 2 supplemental table.

Short title: Minimal change nephrotic syndrome and acute kidney injury

Key words: minimal change nephrotic syndrome, acute kidney injury, hemodialysis, histopathology

Corresponding author and address for reprint requests:

Shinya Nakatani, MD, PhD

Department of Metabolism, Endocrinology and Molecular Medicine, Osaka City University

Graduate School of Medicine, 1-4-3 Asahi-machi, Abeno-ku, Osaka, 545-8585, Japan

Tel: +81-6-6645-3806, Fax: +81-6-6645-3808

Email: m2026719@med.osaka-cu.ac.jp 


\section{Abstract}

Background Acute kidney injury (AKI) is a common complication of minimal change nephrotic syndrome (MCNS), particularly in adults. To predict development of AKI, as defined by the Kidney Disease Improving Global Outcomes classification, we investigated clinical and histopathological features of adult-onset MCNS patients.

Methods A retrospective study was conducted with biopsy-proven adult-onset MCNS patients treated with corticosteroids.

Results A total of 58 MCNS patients [49 (24-71) years old, 38 males] were diagnosed using kidney biopsy findings from 2005 to 2018 at Osaka City University Hospital, of whom 24 (41.4\%) were found to be complicated with AKI. Age, urinary protein, increased body weight (difference from admission to discharge), and histopathological scores were significantly greater in patients with as compared to without AKI, while urinary protein, increased body weight, and interstitial edema score were significantly associated with AKI development [OR: 1.55 (95\% CI 1.04-2.31), 1.37 (95\% CI 1.03-1.81), 20.7 (95\% CI 1.76-243), respectively]. Of the 24 MCNS patients with AKI, 10 underwent transient hemodialysis treatment. Although histopathological features were not different, the time interval between disease onset and kidney biopsy was significantly longer for MCNS patients complicated with AKI requiring hemodialysis as compared to those for whom that was not required [32 (24-46) vs. $13(10-23)$ days, $\mathrm{p}=0.034]$.

Conclusion These results indicate that urinary protein, increased body weight, and interstitial edema score are important information for predicting development of AKI in adult-onset MCNS patients. 


\section{Introduction}

Minimal change nephrotic syndrome (MCNS) refers to the occurrence of nephrotic syndrome histopathologically with normal glomeruli appearance in light microscopy findings, negative immunofluorescence, and foot-process fusion shown by electron microscopy and is the main cause of idiopathic nephrotic syndrome in children [1]. In adults, MCNS affects approximately $10-15 \%$ of patients with idiopathic nephrotic syndrome [2], though is not exceptional in adults older than 65 years $[3,4]$.

Acute kidney injury (AKI) in the context of nephrotic syndrome is a representative clinical complication [5], as it is associated with increased frequency of kidney failure and mortality [6, 7]. Among various nephrotic diseases, AKI is frequently complicated in MCNS cases and reported to occur in approximately $30 \%$ of adult patients with MCNS [8, 9]. AKI is also known to be associated with significantly elevated morbidity and mortality in patients with MCNS [10], thus investigation of factors associated with its development in those cases is considered to be of critical clinical importance.

Previous studies have found that adult-onset MCNS patients complicated with AKI were more likely to be older, male, and affected by hypertension or severe proteinuria $[8,11]$. Acute tubular injury has been also shown in MCNS patients complicated with AKI [8]. However, AKI diagnosis in that study was based on the classical Risk, Injury, Failure, Loss, End-stage kidney disease (RIFLE) classification, originally defined in 2004 [12]. Thereafter, the Kidney Disease Improving Global Outcomes (KDIGO) group assembled all available evidence to form clinical practice guidelines for AKI and proposed the KDIGO classification in 2012 [13]. Presently, diagnosis of AKI using that classification is considered to be more useful for predicting survival outcome as compared to the RIFLE classification [14].

Using AKI as defined by the KDIGO classification, urinary protein was not different 
between adult-onset MCNS patients complicated with AKI and those who were not, though those with AKI showed older age [9, 15], male gender [15], and hypertension [16, 17], while urinary protein was not different between patients with and without AKI. Thus, clinical characteristics associated with AKI in adult-onset MCNS patients remain controversial and detailed information regarding histopathological features in adult-onset MCNS patients complicated with AKI has not been presented. Furthermore, there is scant information regarding the long-term prognosis of AKI patients who have been treated with corticosteroid therapy.

To investigate clinical and histopathological factors associated with AKI in MCNS patients, we performed retrospective clinical and histopathological analyses of 58 biopsy-proven primary adult-onset MCNS cases. 


\section{Materials and Methods}

\section{Patients}

Examinations of pathology registries $(n=1025)$ from May 1, 2005 to October 30, 2018 identified 70 adolescent and adult patients (aged $\geq 15$ years at biopsy) diagnosed with MCNS at Osaka City University Hospital. Excluded were seven because corticosteroid therapy had been administrated prior to the kidney biopsy, two who were complicated with malignancy, two with spontaneous remission, and one for an inadequate biopsy specimen (Fig. 1).

Medical records from electronic charts were reviewed to obtain clinical data from presentation of the disease until the latest follow-up examination. Demographics, presenting characteristics, time (days) from onset of leg pitting edema to kidney biopsy or corticosteroid therapy, medical history, AKI development, hemodialysis information, kidney biopsy histopathological features, and laboratory parameters were collected.

\section{Definitions}

For the present study, we reviewed the complete histopathology of conditions related to kidney biopsy results. Histopathological criteria previously described for MCNS were used, including normal appearing glomeruli shown by light microscopy and absence of detectable immunofluorescence staining for immunoglobulin (Ig) A, IgG, IgM, C1q, and C3 [8].

Evaluation of AKI during therapy was based on the KDIGO classification [13], and defined as an increase in serum creatinine level by $\geq 0.3 \mathrm{mg} / \mathrm{dL}$ within 48 hours or $\geq 1.5$ times the baseline value known or presumed to have occurred within the prior 7 days. Although the KDIGO guidelines also define a urine output classification, we did not use that because hourly urine volume was not measured in any of the enrolled patients, the same as in previous studies [15-17]. The onset of MCNS was defined as the day leg pitting edema was noted. 


\section{Blood sampling}

Laboratory values were determined on the day of the kidney biopsy. All laboratory measurements were performed using routine assays with a standard laboratory method at the Central Clinical Laboratory of the Osaka City University Hospital. Estimated glomerular filtration rate (eGFR) was calculated using the new Japanese coefficient for the abbreviated Modification of Diet in Renal Disease Study equation, including a correction factor of 0.739 for females [18].

\section{Evaluation and histopathological grades of kidney biopsy specimens}

To quantify histopathological alterations, periodic acid-Schiff (PAS)-stained slides were scored by two blinded independent observers (K.N., S.N.). Acute tubular injury shown by kidney biopsy findings was defined by the presence of tubular simplification, loss of brush border, and enlarged reparative nuclei with nucleoli, with or without mitotic figures. Acute tubular injury, tubular atrophy, interstitial edema, and interstitial fibrosis were graded semi-quantitatively on a scale from 0 to 3 on the basis of the percentage of the affected cortical area (Grade $0:<1 \%$, Grade 1: 1-25\%, Grade 2: 26-50\%, Grade 3: >50\%), as previously described [8]. Vascular fibrous intimal thickening was graded from 0 to 3 (absent, mild, moderate, severe, respectively) on the basis of the degree of luminal narrowing and vessel wall thickening [8].

\section{Treatment}

Corticosteroid dose and duration of therapy varied in each patient. However, the initial high-dose corticosteroid therapy usually consisted of prednisone at a dose of 0.6 to $1 \mathrm{mg} / \mathrm{kg}$ daily up to a maximum of $60 \mathrm{mg}$. Tapering was based on improvements of symptoms and proteinuria [5]. Hemodialysis was initiated after group discussion that included all nephrologists in our department, who agreed that it was necessary as relief from excessive fluid, solute, or high degree 
of uremia.

\section{Statistical analyses}

Statistical analyses were performed using Graphpad Prism, version 6.0, for Windows (Graphpad Software, San Diego, CA), and EZR (Saitama Medical Center, Jichi Medical University, Saitama, Japan), a graphical interface for R (The R Foundation for Statistical Computing, Vienna, Austria). Values for clinical characteristics are expressed as the median and interquartile range (IQR), while histopathological scores are expressed as the mean $\pm \mathrm{SD}$. Comparisons between MCNS patients with and without AKI were performed using an unpaired Mann-Whitney $U$ test for continuous variables and a chi-squared test for categorical variables. Comparison of eGFR between the time of the kidney biopsy and one year after corticosteroid therapy was performed using a Wilcoxon rank-sum test. For all patients, factors associated with development of AKI were evaluated by multiple logistic regression analyses. Odds ratio (OR) (95\% confidence interval: $\mathrm{CI}$ ) and $P$ values are described according to the results, with $P$ values $<0.05$ considered to indicate significance. 


\section{Results}

\section{Clinical characteristics of patients with adult-onset MCNS}

The clinical characteristics of 58 MCNS patients at the time of kidney biopsy are presented in Table 1. Age was 49 (24-71) years. Serum creatinine, eGFR, and serum albumin levels were $0.95(0.74-1.10) \mathrm{mg} / \mathrm{dL}, 68.6(47.9-91.9) \mathrm{mL} / \mathrm{min} / 1.73 \mathrm{~m}^{2}$, and $1.80(1.50-2.10) \mathrm{g} / \mathrm{dL}$, respectively, while urinary protein was $6.59(3.95-10.1) \mathrm{g}$ /day. All patients met the criteria for nephrotic syndrome.

\section{Comparisons of clinical characteristics of adult-onset MCNS patients with and without} acute kidney injury

AKI was complicated in $24(41.4 \%)$ patients during the clinical course. According to the KDIGO classification, 9 patients (37.5\%) were Stage 1, 3 (12.5\%) were Stage 2, and $12(50 \%)$ were Stage 3. As shown in Table 1, age [69 (47-75) vs. 31 (20-55) years, p <0.001], body weight change from admission to discharge [13.0 (11.6-16.8) vs. $8.73(5.91-11.5) \mathrm{kg}, \mathrm{p}<0.001]$, and urinary protein levels $[9.59(7.34-11.4)$ vs. $4.76(2.25-6.76) \mathrm{g} /$ day, $\mathrm{p}<0.001]$ were significantly higher in MCNS patients with as compared to those without AKI.

\section{Comparisons of histopathological scores of adult-onset MCNS patients with and without} acute kidney injury

Acute tubular injury was slight in 31 , moderate in 12 , and severe in 5 patients, while tubular atrophy was slight in 27 , moderate in 8 , and severe in 4 , interstitial edema was slight in 18 , moderate in 10 , and severe in 1 , interstitial fibrosis was slight in 16 , moderate in 9 , and severe in 5 , and vascular fibrous intimal thickening was mild in 16 , moderate in 11 , and severe in 5 . Among the 58 kidney biopsy specimens obtained, 5 showed little evidence of acute tubular injury, tubular 
atrophy, interstitial edema, interstitial fibrosis, or vascular fibrous intimal thickening. In MCNS patients with AKI, scores for acute tubular injury, tubular atrophy, interstitial edema, interstitial fibrosis and vascular fibrous intimal thickening were significantly higher, indicating greater severity as compared to those without AKI (Table 1, Fig. 2). Among these histopathological scores, those for acute tubular injury and interstitial edema were significantly associated with development of AKI [OR: 4.48 (95\% CI, 1.07-18.7) and OR: 6.82 (95\% CI, 1.47-31.8), respectively] (Supplemental Table 1).

\section{Association of various clinical and histopathological parameters with AKI}

Next, the association of clinical and histopathological parameters with development of AKI was examined. Using multiple logistic regression analysis to explore factors associated with development of AKI, age, gender, systolic blood pressure, urinary protein, increased body weight (difference from admission to discharge), and histopathological interstitial edema score were included as explanatory variables. Since interstitial edema was the strongest factor associated with AKI development among the five histopathological scores, it was used as a histopathological factor in multiple logistic regression analysis (Supplemental Table 1). The results showed that gender, urinary protein, increased body weight, and histopathological interstitial edema score were independently associated with development of AKI. The OR values for the association of urinary protein, increased body weight and interstitial edema score with development of AKI

were 1.55 (95\% CI, 1.04-2.31), 1.37 (95\% CI, 1.03-1.81) and 20.7 (95\% CI, 1.76-243), respectively (Table 2).

\section{Comparison of clinical characteristics and histopathological scores of adult-onset MCNS patients with AKI with and without required hemodialysis}


Of 24 MCNS patients with AKI, 10 (41.7\%) required transient hemodialysis treatment, which was performed 1 to 54 times, ranging from 3 to 132 days. The age of MCNS patients with AKI requiring hemodialysis was significantly higher than that of those who did not require that treatment. Also, kidney function parameters in the former were significantly worse as compared to those in the latter [eGFR: $26.7(16.9-39.1)$ vs. $59.5(39.7-73.0) \mathrm{ml} / \mathrm{min} / 1.73 \mathrm{~m}^{2}, \mathrm{p}=0.030$; serum creatinine: $2.17(1.06-3.00)$ vs. $0.86(0.68-1.44) \mathrm{mg} / \mathrm{dL}, \mathrm{p}=0.014]$ (Table 3). Urinary protein, serum albumin levels, histopathological scores, and the time interval from onset of leg pitting edema to onset of AKI were not significantly different between the two groups. Time interval to kidney biopsy from onset of leg pitting edema was significantly longer for patients who required hemodialysis [32 (24-46) vs. $13(10-23)$ days, $\mathrm{p}=0.034]$ (Table 3).

Regarding other treatments, angiotensin-converting enzyme inhibitor (ACEI) / angiotensin receptor blocker (ARB), non-steroidal anti-inflammatory drug, and diuretics usage from the onset of leg pitting edema to onset of AKI were not significantly different between the two groups.

\section{Kidney function during clinical course in MCNS patients}

Table 4 shows changes in eGFR at the time of the kidney biopsy and at follow-up examinations performed for up to 1 year after beginning corticosteroid therapy. Among the 58 enrolled MCNS patients, follow-up data for 45 were available. In those without AKI $(n=26)$, eGFR did not show a significant change during the follow-up period [81.8 (68.1-94.9) and 76.8 (73.3-97.6) $\mathrm{mL} / \mathrm{min} / 1.73 \mathrm{~m}^{2}$ at the kidney biopsy and 1-year follow-up examination, respectively]. Although eGFR was lower than $60 \mathrm{~mL} / \mathrm{min} / 1.73 \mathrm{~m}^{2}$ in two patients at 1 year after starting corticosteroid therapy ( 48.3 and $50.2 \mathrm{~mL} / \mathrm{min} / 1.73 \mathrm{~m}^{2}$, respectively), that at the time of the kidney biopsy was already reduced (38.9 and $48.0 \mathrm{~mL} / \mathrm{min} / 1.73 \mathrm{~m}^{2}$, respectively).

In those with AKI (n=19), eGFR was improved during the follow-up period, though not 
significantly $(\mathrm{p}=0.134)$, as it was $55.8(23.0-71.5)$ and $66.3(47.1-76.6) \mathrm{mL} / \mathrm{min} / 1.73 \mathrm{~m}^{2}$ at the time of the kidney biopsy and 1-year follow-up examination, respectively. In AKI patients who did not undergo hemodialysis $(\mathrm{n}=10)$, eGFR were not significantly different at those two examination time points [65.3 (59.5-84.1) and $75.2(67.6-76.8) \mathrm{mL} / \mathrm{min} / 1.73 \mathrm{~m}^{2}$, respectively]. In patients with AKI requiring hemodialysis treatment $(n=9)$, hemodialysis was successfully withdrawn in all cases within 132 days, with eGFR significantly improved from 25.4 (15.7-40.2) to $44.4(38.6-58.4) \mathrm{mL} / \mathrm{min} / 1.73 \mathrm{~m}^{2}(\mathrm{p}=0.027)$. 


\section{Discussion}

In this retrospective study of 58 patients with adult-onset MCNS, AKI defined by the KDIGO classification was noted in $41.4 \%(n=24)$ during the clinical course. Higher urinary protein, increased body weight (difference from admission to discharge), and greater interstitial edema score were independently associated with AKI development. Ten of the 24 MCNS patients with AKI required hemodialysis, with older age and more severe kidney dysfunction significant in those cases. Interestingly, the time interval to kidney biopsy from the onset of leg pitting edema was significantly longer in AKI patients requiring hemodialysis as compared to those who did not require that. Our findings indicate that urinary protein, increased body weight and interstitial edema score are important information for predicting development of AKI in patients with adultonset MCNS. Additionally, a shorter time to kidney biopsy may be important to avoid hemodialysis in these cases.

The proportion of AKI cases (41.4\%) in the present cohort was slightly higher as compared to the four previous studies [9, 15-17], while the age range [69 (47-75) years] of MCNS patients with AKI in our study was also higher as compared to those prior reports (44-53 years). Other clinical parameters, such as blood pressure, serum albumin, and urinary protein levels were not different, between the present and previous studies. Older age is known to be a risk factor for AKI in patients with adult-onset MCNS $[8,9,11,15]$. Accordingly, the greater age of the present cohort may have contributed to the higher rate of AKI.

The present study is the first to demonstrate that a significantly greater increase in body weight is related with development of AKI in MCNS cases. The underlying mechanisms of AKI in these patients remain unclear, as the traditional "underfill" hypothesis as well as reduction in effective circulating blood volume in the kidneys are no longer believed responsible for reduced GFR in MCNS patients [10]. Instead, ischemic tubular injury [8, 10] and vasoconstriction [19] 
are currently emphasized, while the nephrosarca hypothesis, which states that interstitial edema of the kidneys in MCNS patients results in increased hydrostatic pressure in the proximal tubule and Bowman's space, leading to a decrease in GFR [20,21], has also been proposed. In the present study, histopathological interstitial edema was found to be independently associated with AKI development. Thus, increased body fluid reflected by increased body weight may lead to interstitial edema in the kidneys, possibly resulting in development of AKI in MCNS cases. Patients receiving care in our department undergo routine body weight measurements every morning, because it is non-invasive and easy to perform as compared to laboratory examinations. The present findings strongly support the importance of evaluation of body weight in the clinical setting of MCNS patient care.

A few studies have presented histopathology results of kidney biopsy specimens from MCNS patients complicated with AKI defined by either the traditional [8] or KDIGO [17] classification. Histopathological alterations of tubules were emphasized in those reports, but not glomerular abnormalities. In those previous studies, tubular necrosis and tubular simplification were significantly worse and more severe in MCNS patients with AKI as compared to those without, whereas severe interstitial edema with tubular collapse, fibrosis, and atherosclerosis or arteriosclerosis were not different between those two groups [17], while among 22 MCNS patients with AKI, features of those affected by acute tubular injury $(n=14)$, interstitial edema $(n=9)$, mild tubular atrophy and interstitial fibrosis $(n=13)$, and atherosclerosis $(n=15)$ were emphasized [8]. In the present cohort, histopathological scores for acute tubular injury, tubular atrophy, interstitial edema, interstitial fibrosis, and vascular fibrous intimal thickening were significantly higher in adult-onset MCNS patients with AKI as compared to those without. Among the histopathological scores examined, acute tubular injury and interstitial edema were significantly associated with development of AKI (Supplemental Table 1). The findings of the present study as well as previous 
studies indicate that evaluations of tubule-interstitial alterations $[8,17,19]$, especially acute tubular injury and interstitial edema, are important for prediction of development of AKI in MCNS patients.

Hemodialysis treatment is frequently necessary for adult-onset MCNS patients. In a Medline search covering a recent 25-year period (1993-2017), Meyrier A, et al. identified 13 related publications that presented 235 (33\%) of 716 patients of adult-onset MCNS complicated with AKI. Among those 235 cases, 52 (25\%) required treatment with dialysis for periods ranging from a few days to several months [10]. In the present study, hemodialysis was noted in 10 (41.7\%) of 24 MCNS patients complicated with AKI, a slightly higher incidence as compared to that previous analysis. The higher incidence of hemodialysis shown in our study may have been due to the older age of the enrolled cohort, as 8 of 10 for whom hemodialysis treatment was performed were older than 70 years. The hemodialysis treatment period for all of those in our study ranged from 3 to 132 days and none required hemodialysis treatment at the most recent follow-up examination. However, an arteriovenous fistula was noted in 3 patients, which can impact quality of life, and is also a risk factor for infection and congestive heart failure [22], particularly in elderly individuals. Thus, it is important to investigate clinical factors associated with the requirement for hemodialysis. In the present study, the time interval between disease onset and start of corticosteroid therapy was significantly longer for adult-onset MCNS patients complicated with AKI requiring hemodialysis as compared to those for whom that was not required. Earlier kidney biopsy from MCNS onset might be important to avoid hemodialysis treatment for MCNS patients complicated with AKI. Additional studies from a wide range of institutions are needed to confirm our tentative results regarding this matter.

This study has some limitations. First, it was conducted as a retrospective single-center investigation with a relatively small sample size. Second, we were not able to completely rule out 
the existence of a pre-kidney factor related to AKI, such as dehydration or administration of drugs harmful to the kidneys. Although the long-term protective effects of ACEI / ARB administration in patients with proteinuria have been investigated, their use may contribute to development of AKI [23]. In the present study, 10 (17.2\%) patients were treated with ACEI / ARB and $14(24.1 \%)$ with diuretics prior to onset of leg pitting edema. Furthermore, to maintain urine volume during the clinical course, diuretics were added in $25(43.1 \%)$ of the cases after onset of leg pitting edema. There was no independent association with AKI development noted for ACEI / ARB, diuretics, or non-steroidal anti-inflammatory drug administration (Supplemental Table 2).

\section{Conclusion}

In summary, this is the first study to report statistical findings of adult-onset MCNS patients with AKI defined by KDIGO classification, which were obtained with simultaneous analyses of both clinical characteristics and tubule-interstitial alterations shown by kidney biopsy results. Those results indicate that urinary protein, increased body weight, and histopathological scoring showing interstitial edema are independently associated with development of AKI in adult-onset MCNS patients.

\section{Acknowledgements}

We thank Drs. Rino Nakaya, Minenori Ichikawa, Ayumi Shuto, Kazuma Sone, Yusuke Fukuda, Kenta Fujimoto, Ayumi Nakatani, Mari Sakura, Yujiro Okute, Hisanori Okazaki, Mio Toyokawa, Mika Sonoda, Mitsuru Ichii, Yoshiteru Ohno, Hideaki Shima, Ikue Kobayashi, Yu Tateishi, Hirokazu Okazaki, Kaori Shidara, Kaname Hirowatari, Takao Tsuchida, and Naoki Matsumoto for their assistance in collecting samples and providing patient care. We also thank 
Professor Masahiko Osawa, MD, PhD, of the Department of Diagnostic Pathology, Osaka City University Graduate School of Medicine, for assistance with the histopathological examinations.

\section{Authors' contributions}

K.N. contributed to study concept and design, data acquisition, analyses, interpretation, and writing the manuscript. S.N. and E.I. contributed to study concept and design, analyses, interpretation, and writing the manuscript. F.M., Y.M., H.U., A.T., K.M., M.I., and M.E. contributed to study concept and data interpretation. All authors have read and approved the submitted manuscript.

\section{Compliance with ethical standards}

\section{Conflict of interest}

None of the authors have conflicts of interests to declare in regard to this study.

\section{Ethical approval}

The Institutional Review Board of Osaka City University Graduate School of Medicine approved the use of kidney biopsy specimens and clinical data in accordance with the Declaration of Helsinki and guidelines of the Osaka City University Graduate School of Medicine (approval no. 2378).

\section{Informed consent}


Informed consent for the kidney biopsy procedure was obtained from all individual patients included in the study. An opt-out option was given, as explained in instructions posted on the website of the institution.

\section{References}

1. Eddy AA, Symons JM. Nephrotic syndrome in childhood. Lancet. 2003;362:629-39.

2. Jefferson JA, Nelson PJ, Najafian B, Shankland SJ. Podocyte disorders: Core Curriculum 2011. Am J Kidney Dis. 2011;58:666-77.

3. Uezono S, Hara S, Sato Y, Komatsu H, Ikeda N, Shimao Y et al. Renal biopsy in elderly patients: a clinicopathological analysis. Ren Fail. 2006;28:549-55.

4. Yokoyama H, Sugiyama H, Sato H, Taguchi T, Nagata M, Matsuo S et al. Renal disease in the elderly and the very elderly Japanese: analysis of the Japan Renal Biopsy Registry (J-RBR).

Clin Exp Nephrol. 2012;16:903-20.

5. Nishi S, Ubara Y, Utsunomiya Y, Okada K, Obata Y, Kai H et al. Evidence-based clinical practice guidelines for nephrotic syndrome 2014. Clin Exp Nephrol. 2016;20:342-70.

6. Chertow GM, Burdick E, Honour M, Bonventre JV, Bates DW. Acute kidney injury, mortality, length of stay, and costs in hospitalized patients. J Am Soc Nephrol. 2005;16:3365-70. 7. Silvi Shah, Anthony C. Leonard, Kathleen Harrison, Karthikeyan Meganathan, Annette L. Christianson and Charuhas V. Thakar. Mortality and Recovery Associated with Kidney Failure due to Acute Kidney Injury. Clin J Am Soc Nephrol. 2020;15:995-1006.

8. Waldman M, Crew RJ, Valeri A, Busch J, Stokes B, Markowitz G et al. Adult minimalchange disease: clinical characteristics, treatment, and outcomes. Clin J Am Soc Nephrol. 2007;2:445-53.

9. Fenton A, Smith SW, Hewins P. Adult minimal-change disease: observational data from a UK centre on patient characteristics, therapies, and outcomes. BMC Nephrol. 2018;19:207.

10. Meyrier A, Niaudet P. Acute kidney injury complicating nephrotic syndrome of minimal change disease. Kidney Int. 2018;94:861-9.

11. Maas RJ, Deegens JK, Beukhof JR, Reichert LJ, Ten dam MA, Beutler JJ et al. The clinical course of minimal change nephrotic syndrome with onset in adulthood or late adolescence: a case series. Am J Kidney Dis. 2017;69:637-46.

12. Bellomo R, Ronco C, Kellum JA, Mehta RL, Palevsky P, Acute Dialysis Quality Initiative w. Acute renal failure - definition, outcome measures, animal models, fluid therapy and information technology needs: the Second International Consensus Conference of the Acute 
Dialysis Quality Initiative (ADQI) Group. Crit Care. 2004;8:R204-12.

13. Khwaja A. KDIGO clinical practice guidelines for acute kidney injury. Nephron Clin Pract. 2012;120:c179-84.

14. Doi K, Nishida O, Shigematsu T, Sadahiro T, Itami N, Iseki K et al. The Japanese Clinical Practice Guideline for acute kidney injury 2016. J Intensive Care. 2018;6:48.

15. Huang MJ, Wei RB, Su TY, Wang Y, Li QP, Yang X et al. Impact of acute kidney injury on coagulation in adult minimal change nephropathy. Medicine (Baltimore). 2016;95:e5366.

16. Komukai D, Hasegawa T, Kaneshima N, Takayasu M, Sato Y, Hirose M et al. Influence of acute kidney injury on the time to complete remission in adult minimal change nephrotic syndrome: a single-centre study. Nephrology (Carlton). 2016;21:887-92.

17. Fujigaki Y, Tamura Y, Nagura M, Arai S, Ota T, Shibata $S$ et al. Unique proximal tubular cell injury and the development of acute kidney injury in adult patients with minimal change nephrotic syndrome. BMC Nephrol. 2017;18:339.

18. Matsuo S, Imai E, Horio M, Yasuda Y, Tomita K, Nitta K et al. Revised equations for estimated GFR from serum creatinine in Japan. Am J Kidney Dis. 2009;53:982-92.

19. Chen CL, Fang HC, Chou KJ, Lee JC, Lee PT, Chung HM et al. Increased endothelin 1 expression in adult-onset minimal change nephropathy with acute renal failure. Am J Kidney Dis. 2005;45:818-25.

20. Lowenstein J, Schacht RG, Baldwin DS. Renal failure in minimal change nephrotic syndrome. Am J Med. 1981;70:227-33.

21. Oyama Y, Iwafuchi Y, Morioka T, Narita I. Acute Kidney Injury Associated with Minimal Change Nephrotic Syndrome in an Elderly Patient Successfully Treated with both Fluid Management and Specific Therapy Based on Kidney Biopsy Findings. Case Rep Nephrol Dial. 2020;10:42-50.

22. Stolic R. Most important chronic complications of arteriovenous fistulas for hemodialysis. Med Princ Pract. 2013;22:220-8.

23. Kuriyama S, Sugano N, Ueda H, Otsuka Y, Kanzaki G, Hosoya T. Successful effect of triple blockade of renin-angiotensin-aldosterone system on massive proteinuria in a patient with chronic kidney disease. Clin Exp Nephrol. 2009;13:663-6. 


\begin{tabular}{|c|c|c|c|c|}
\hline & All patients & $\begin{array}{l}\text { MCNS with } \\
\text { AKI }\end{array}$ & $\begin{array}{l}\text { MCNS without } \\
\text { AKI }\end{array}$ & P value \\
\hline Male / female, $\mathrm{n}$ & $38 / 20$ & $13 / 11$ & $25 / 9$ & 0.165 \\
\hline Age, years & $49(24-71)$ & $69(47-75)$ & $31(20-55)$ & $<0.001$ \\
\hline Body weight at admission, $\mathrm{kg}$ & $64.2(56.6-74.2)$ & $62.0(55.0-67.6)$ & $67.5(61.0-76.5)$ & 0.064 \\
\hline BMI at admission, $\mathrm{kg} / \mathrm{m}^{2}$ & $23.9(21.9-26.7)$ & $23.8(22.1-26.1)$ & $23.9(21.9-27.7)$ & 0.857 \\
\hline Body weight at discharge, $\mathrm{kg}$ & $54.6(46.6-61.7)$ & $46.8(42.9-53.4)$ & $58.1(53.1-66.4)$ & $<0.001$ \\
\hline BMI at discharge, $\mathrm{kg} / \mathrm{m}^{2}$ & $19.3(18.3-22.5)$ & $18.5(17.2-19.5)$ & $21.2(19.1-23.8)$ & 0.001 \\
\hline $\begin{array}{l}\text { Body weight change from admission to } \\
\text { discharge, } \mathrm{kg}\end{array}$ & $11.2(7.7-13.4)$ & $13.0(11.6-16.8)$ & $8.73(5.91-11.5)$ & $<0.001$ \\
\hline Systolic blood pressure, $\mathrm{mmHg}$ & $120(105-134)$ & $128(119-138)$ & $110(104-124)$ & 0.008 \\
\hline Diastolic blood pressure, $\mathrm{mmHg}$ & $70(60-78)$ & $76(69-86)$ & $64(58-74)$ & 0.002 \\
\hline $\mathrm{CRP}, \mathrm{mg} / \mathrm{dL}$ & $0.07(0.02-0.17)$ & $0.12(0.05-0.20)$ & $0.05(0.01-0.15)$ & 0.093 \\
\hline Serum total protein, $\mathrm{g} / \mathrm{dL}$ & $4.35(3.90-4.78)$ & $4.25(3.88-4.73)$ & $4.40(4.10-4.85)$ & 0.444 \\
\hline Serum albumin, $\mathrm{g} / \mathrm{dL}$ & $1.80(1.50-2.10)$ & $1.70(1.50-2.03)$ & $1.90(1.43-2.20)$ & 0.406 \\
\hline Blood urea nitrogen, $\mathrm{mg} / \mathrm{dL}$ & $16.0(13.3-25.8)$ & $30.0(15.8-44.3)$ & $15.0(12.3-17.0)$ & 0.001 \\
\hline Serum creatinine, $\mathrm{mg} / \mathrm{dL}$ & $0.95(0.74-1.10)$ & $1.12(0.74-2.38)$ & $0.89(0.75-0.98)$ & $<0.001$ \\
\hline $\mathrm{eGFR}, \mathrm{mL} / \mathrm{min} / 1.73 \mathrm{~m}^{2}$ & $68.6(47.9-91.9)$ & $44.0(24.2-63.7)$ & $84.7(68.1-98.2)$ & $<0.001$ \\
\hline Urinary protein, g/day & $6.59(3.95-10.1)$ & $9.59(7.34-11.4)$ & $4.76(2.25-6.76)$ & $<0.001$ \\
\hline $\begin{array}{l}\text { Time to kidney biopsy from onset of leg } \\
\text { pitting edema, days }\end{array}$ & $15(9-29.5)$ & $20(11-33)$ & $14(8-26)$ & 0.435 \\
\hline $\begin{array}{l}\text { Time to corticosteroid therapy from onset of } \\
\text { leg pitting edema, days }\end{array}$ & $23(14-35)$ & $27(14-36)$ & $20(14-34)$ & 0.586 \\
\hline Initial corticosteroid dosage, $\mathrm{mg} /$ day & $48(40-50)$ & $40(40-50)$ & $50(40-50)$ & 0.025 \\
\hline \multicolumn{5}{|l|}{ Histopathological scoring } \\
\hline Acute tubular injury & $1.21 \pm 0.83$ & $1.79 \pm 0.83$ & $0.79 \pm 0.54$ & $<0.001$ \\
\hline Tubular atrophy & $0.95 \pm 0.87$ & $1.33 \pm 0.87$ & $0.68 \pm 0.77$ & 0.003 \\
\hline Interstitial edema & $0.71 \pm 0.82$ & $1.33 \pm 0.82$ & $0.27 \pm 0.45$ & $<0.001$ \\
\hline Interstitial fibrosis & $0.84 \pm 0.99$ & $1.42 \pm 0.97$ & $0.44 \pm 0.86$ & $<0.001$ \\
\hline Vascular fibrous intimal thickening & $0.91 \pm 1.00$ & $1.29 \pm 1.00$ & $0.65 \pm 0.92$ & 0.008 \\
\hline Presence of hypertension, $\mathrm{n}(\%)$ & $19(32.8 \%)$ & $11(45.8 \%)$ & $8(23.5 \%)$ & 0.094 \\
\hline ACEI / ARB use, n (\%) & $8(13.8 \%)$ & $1(4.2 \%)$ & $7(20.6 \%)$ & 0.123 \\
\hline Diuretic use, n (\%) & $39(67.2 \%)$ & $20(83.3 \%)$ & $19(55.9 \%)$ & 0.046 \\
\hline NSAID use, n (\%) & $3(5.2 \%)$ & $1(4.2 \%)$ & $2(5.9 \%)$ & 1.000 \\
\hline
\end{tabular}

Values are shown as number and percentage for categorical variables or median (IQR) for continuous variables of clinical characteristics. Histopathological scores are expressed as the mean \pm SD. $P$ values were obtained with a $\chi^{2}$ or Mann-Whitney $U$ test for comparisons between MCNS patients with and without AKI. 
Abbreviations: ACEI, angiotensin-converting enzyme inhibitor; AKI, acute kidney injury; ARB, angiotensin receptor blocker; BMI, body mass index; CRP, C-reactive protein; eGFR, estimated glomerular filtration rate; IQR, interquartile range; NSAID, non-steroidal anti-inflammatory drug; MCNS, minimal change nephrotic syndrome; SD, standard deviation 
Table 2 Factors associated with AKI in MCNS patients (multiple logistic regression analysis)

\begin{tabular}{lccc}
\hline Variables & OR & $95 \%$ CI & $P$ value \\
\hline Age $(/$ year $)$ & 0.97 & $0.91-1.03$ & 0.274 \\
Gender $($ female $=0$, male $=1)$ & 0.04 & $0.002-0.700$ & 0.028 \\
Systolic blood pressure $(/ \mathrm{mmHg})$ & 0.97 & $0.91-1.09$ & 0.293 \\
Urinary protein $(/ \mathrm{g} /$ day) & 1.55 & $1.04-2.31$ & 0.031 \\
Body weight change from admission to discharge $(/ \mathrm{kg})$ & 1.37 & $1.03-1.81$ & 0.031 \\
Interstitial edema score $(/$ point $)$ & 20.7 & $1.76-243$ & 0.016 \\
\hline
\end{tabular}

Odds ratio: OR [95\% confidential interval (CI)].

Abbreviations: CI, confidence interval; MCNS, minimal change nephrotic syndrome; OR, odds ratio 
Table 3 Comparisons of clinical characteristics of MCNS patients complicated with AKI with or without hemodialysis requirement

\begin{tabular}{|c|c|c|c|}
\hline & With hemodialysis & Without hemodialysis & $P$ value \\
\hline Male / female, $\mathrm{n}$ & $8 / 2$ & $5 / 9$ & 0.047 \\
\hline Age, years & $75(73-77.8)$ & $54(46-70)$ & 0.043 \\
\hline Body weight at admission, $\mathrm{kg}$ & $62.4(54.5-64.2)$ & $62.0(55.1-72.4)$ & 0.536 \\
\hline BMI at admission, $\mathrm{kg} / \mathrm{m}^{2}$ & $24.0(21.1-26.5)$ & $23.8(22.3-25.6)$ & 0.796 \\
\hline Body weight at discharge, $\mathrm{kg}$ & $45.2(40.7-49.2)$ & $47.2(44.2-55.9)$ & 0.265 \\
\hline $\mathrm{BMI}$ at discharge, $\mathrm{kg} / \mathrm{m}^{2}$ & $18.6(15.8-19.3)$ & $18.5(17.3-20.6)$ & 0.437 \\
\hline $\begin{array}{l}\text { Body weight change from admission to } \\
\text { discharge, } \mathrm{kg}\end{array}$ & $13.0(11.4-15.2)$ & $13.0(11.8-17.3)$ & 0.989 \\
\hline Systolic blood pressure, $\mathrm{mmHg}$ & $138(126-148)$ & $123(118-134)$ & 0.081 \\
\hline Diastolic blood pressure, $\mathrm{mmHg}$ & $74(68-85)$ & $77(70-85)$ & 0.966 \\
\hline $\mathrm{CRP}, \mathrm{mg} / \mathrm{dL}$ & $0.16(0.12-0.38)$ & $0.07(0.02-0.14)$ & 0.036 \\
\hline Serum total protein, $\mathrm{g} / \mathrm{dL}$ & $4.05(3.90-4.38)$ & $4.40(3.78-4.78)$ & 0.655 \\
\hline Serum albumin, $\mathrm{g} / \mathrm{dL}$ & $1.65(1.35-1.98)$ & $1.75(1.63-2.05)$ & 0.444 \\
\hline Blood urea nitrogen, $\mathrm{mg} / \mathrm{dL}$ & $44.5(29-48.3)$ & $16.0(12.0-32.8)$ & 0.003 \\
\hline Serum creatinine, $\mathrm{mg} / \mathrm{dL}$ & $2.17(1.06-3.00)$ & $0.86(0.68-1.44)$ & 0.014 \\
\hline $\mathrm{eGFR}, \mathrm{mL} / \mathrm{min} / 1.73 \mathrm{~m}^{2}$ & $26.7(16.9-39.1)$ & $59.5(39.7-73.0)$ & 0.030 \\
\hline Urinary protein, $g /$ day & $10.5(9.27-12.3)$ & $8.33(6.00-10.4)$ & 0.108 \\
\hline $\begin{array}{l}\text { Time to kidney biopsy from onset of leg } \\
\text { pitting edema, days }\end{array}$ & $32(24-46)$ & $13(10-23)$ & 0.034 \\
\hline $\begin{array}{l}\text { Time to corticosteroid therapy from onset of } \\
\text { leg pitting edema, days }\end{array}$ & $34(29-51)$ & $18(13-29)$ & 0.034 \\
\hline $\begin{array}{l}\text { Time to onset of AKI from onset of leg } \\
\text { pitting edema, days }\end{array}$ & $29(7-46)$ & $17(9-21)$ & 0.348 \\
\hline Initial corticosteroid dosage, $\mathrm{mg} /$ day & $40(40-50)$ & $40(40-50)$ & 0.388 \\
\hline \multicolumn{4}{|l|}{ Histopathological scoring of kidney biopsy } \\
\hline Acute tubular injury & $1.90 \pm 0.74$ & $1.71 \pm 0.91$ & 0.668 \\
\hline Tubular atrophy & $1.30 \pm 1.06$ & $1.36 \pm 0.75$ & 0.771 \\
\hline Interstitial edema & $1.60 \pm 0.84$ & $1.14 \pm 0.77$ & 0.199 \\
\hline Interstitial fibrosis & $1.60 \pm 1.17$ & $1.29 \pm 0.83$ & 0.521 \\
\hline Vascular fibrous intimal thickening & $1.60 \pm 1.17$ & $1.07 \pm 0.83$ & 0.286 \\
\hline Presence of hypertension, $\mathrm{n}(\%)$ & $7(70 \%)$ & $4(28.6 \%)$ & 0.095 \\
\hline ACEI / ARB use, n (\%) & $1(10 \%)$ & $0(0 \%)$ & 0.417 \\
\hline Diuretic use, n (\%) & $10(100 \%)$ & $10(71.4 \%)$ & 0.114 \\
\hline NSAID use, n (\%) & $0(0 \%)$ & $1(7.1 \%)$ & 1.000 \\
\hline
\end{tabular}

Values are shown as number and percentage for categorical variables or median (IQR) for continuous variables of clinical characteristics. Histopathological scores are expressed as the mean \pm SD. $P$ values were obtained with a $\chi^{2}$ or 
Mann-Whitney $U$ test for comparison between MCNS patients who did and did not require hemodialysis.

Abbreviations: ACEI, angiotensin-converting enzyme inhibitor; AKI, acute kidney injury; ARB, angiotensin receptor blocker; BMI, body mass index; CRP, C-reactive protein; eGFR, estimated glomerular filtration rate; IQR, interquartile range; NSAID, non-steroidal anti-inflammatory drug; MCNS, minimal change nephrotic syndrome; SD, standard deviation 
Table 4 Comparison of eGFR at kidney biopsy and at one-year follow-up examination performed in 45 of 58 MCNS patients

\begin{tabular}{lccc}
\hline & $\begin{array}{c}\text { Time of kidney } \\
\text { biopsy }\end{array}$ & One year later & $P$ value \\
\hline All patients, $\mathrm{n}=45$ & $68.6(50.2-90.0)$ & $75.3(60.7-88.6)$ & 0.056 \\
Without AKI, $\mathrm{n}=26$ & $81.8(68.1-94.9)$ & $76.8(73.3-97.6)$ & 0.291 \\
With AKI, $\mathrm{n}=19$ & $55.8(23.0-71.5)$ & $66.3(47.1-76.6)$ & 0.134 \\
$\quad$ Hemodialysis treatment not required, $\mathrm{n}=10$ & $65.3(59.5-84.1)$ & $75.2(67.6-76.8)$ & 0.922 \\
$\quad$ Hemodialysis treatment required, $\mathrm{n}=9$ & $25.4(15.7-40.2)$ & $44.4(38.6-58.4)$ & 0.027 \\
\hline
\end{tabular}

Values are expressed as the median (IQR).

Abbreviations: AKI, acute kidney injury; MCNS, minimal change nephrotic syndrome 
Supplemental Table 1 Histopathological factors associated with AKI in MCNS patients (multiple logistic regression analysis)

\begin{tabular}{lccc}
\hline Explanatory variables & OR & $95 \%$ CI & $P$ value \\
\hline Acute tubular injury score (/ point) & 4.48 & $1.07-18.7$ & 0.040 \\
Tubular atrophy score (/ point) & 0.80 & $0.10-6.39$ & 0.837 \\
Interstitial edema score (/ point) & 6.82 & $1.47-31.8$ & 0.014 \\
Interstitial fibrosis score (/ point) & 1.06 & $0.13-8.41$ & 0.958 \\
Vascular fibrous intimal thickening score (/ point) & 0.93 & $0.33-2.65$ & 0.898 \\
\hline
\end{tabular}

Odds ratio: OR [95\% confidential interval $(\mathrm{CI})]$.

Abbreviations: AKI, acute kidney injury; CI, confidence interval; MCNS, minimal change nephrotic syndrome; OR, odds ratio

Supplemental Table 2 Factors associated with AKI in MCNS patients (multiple logistic regression analysis)

\begin{tabular}{lccc}
\hline Variables & OR & $95 \%$ CI & $P$ value \\
\hline Age $(/$ year) & 0.98 & $0.91-1.04$ & 0.474 \\
Gender (female = , male = 1) & 0.02 & $0.001-0.893$ & 0.043 \\
Systolic blood pressure (/ mmHg) & 1.03 & $0.97-1.10$ & 0.282 \\
Urinary protein (/ g/day) & 1.62 & $1.04-2.52$ & 0.033 \\
Body weight change from admission to discharge (/ kg) & 1.40 & $1.02-1.91$ & 0.035 \\
Interstitial edema score (/ point) & 20.9 & $1.78-245$ & 0.016 \\
ACEI / ARB and/or diuretics and/or NSAID & 0.30 & $0.02-5.15$ & 0.408 \\
(non-user = 0, user = 1) & & & \\
\hline
\end{tabular}

Odds ratio: OR [95\% confidential interval (CI)].

Abbreviations are the same as in the Table 2 


\section{Figure legends}

Fig. 1 Flowchart of study design

This was a retrospective single-center observational study of biopsy-proven first-onset adult patients with minimal change nephrotic syndrome (MCNS) treated with corticosteroids. There were 24 patients with acute kidney injury (AKI) and 34 without. 


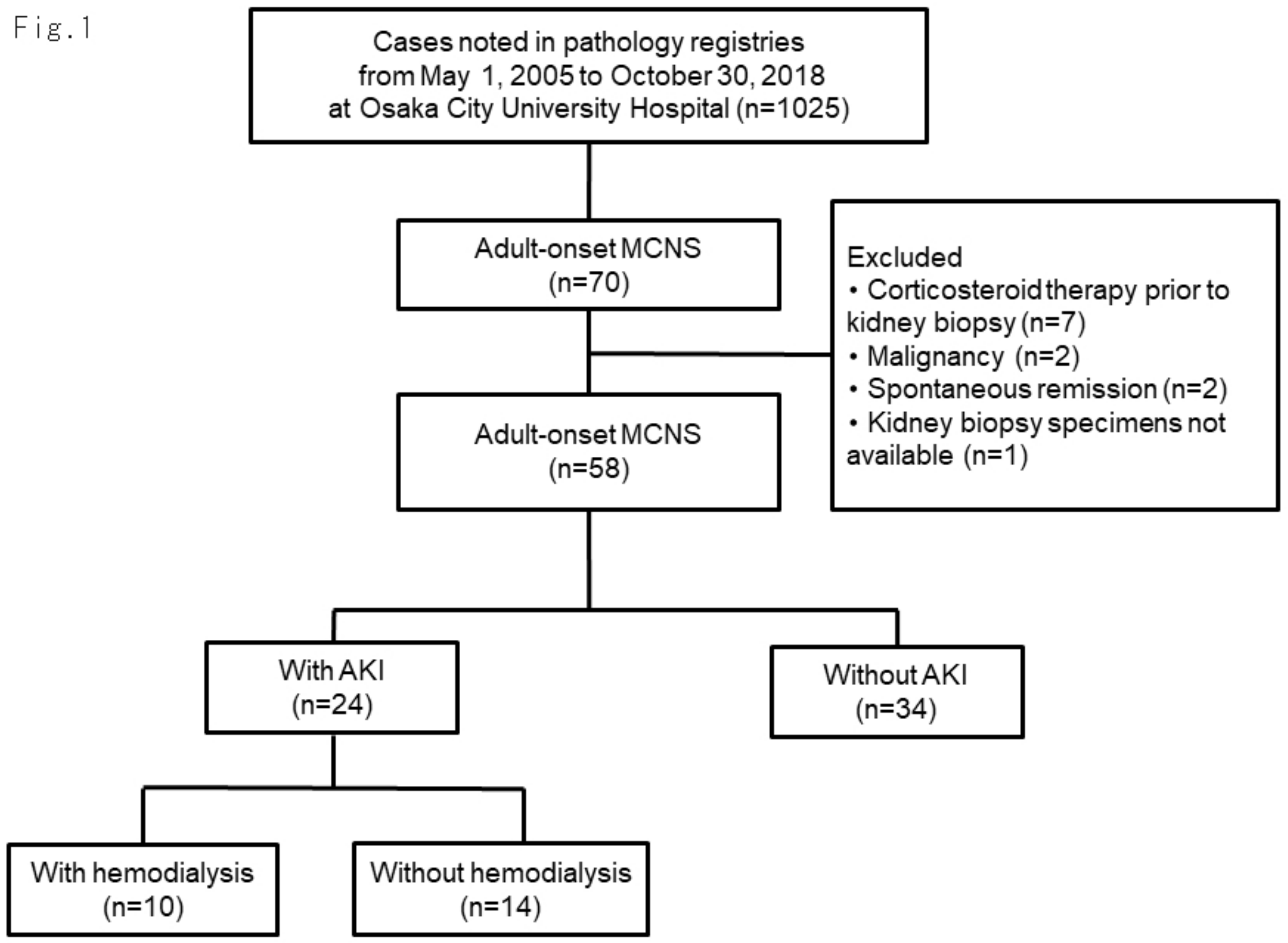


Fig. 2 Comparison of histopathological scores between minimal change nephrotic syndrome (MCNS) patients without and with acute kidney injury $(\mathrm{AKI})$

a) Acute tubular injury, b) tubular atrophy, c) interstitial edema, d) interstitial fibrosis, and e) vascular fibrous intimal thickening scores were significantly worse in MCNS patients with as compared to those without AKI. 
Fig. 2
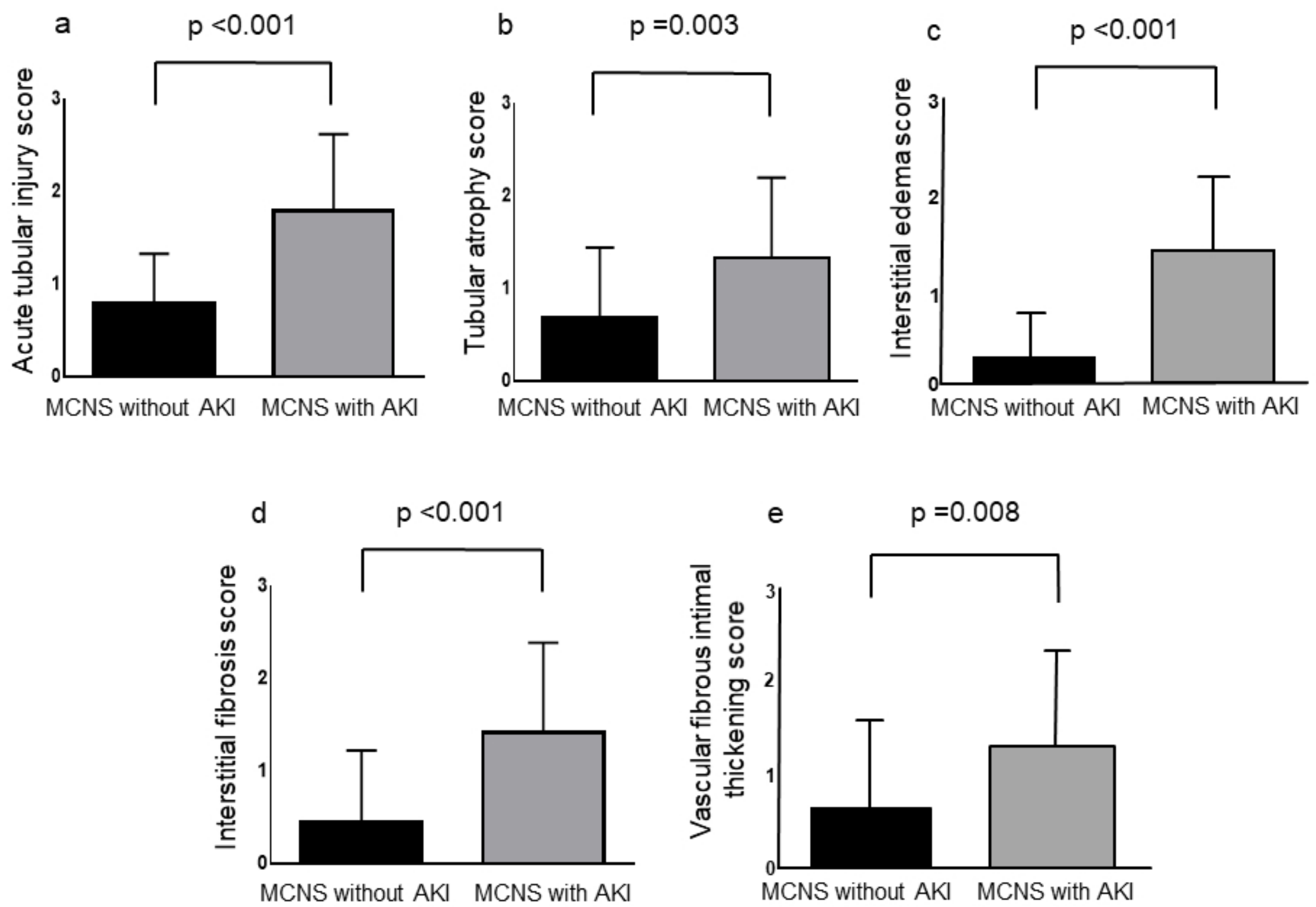